\title{
Actitud del universitario hacia el uso del cannabis como tratamiento del dolor crónico
}

Domingo-Martínez Gabriela Lorena*, Sánchez-Carbajal Karen Daniela*, Ruíz-Vargas Nancy Viridiana**, Gallegos-Torres Ruth Magdalena**, Oñate-Romero Juan Manuel ${ }^{* * *}$, Juárez-Nilo Sarket Gizeh****, Xeque-Morales Ángel****

\section{- Resumen}

- Objetivo: describir la actitud de estudiantes de una universidad pública

- hacia el uso del cannabis como tratamiento del dolor crónico, con la

- finalidad de establecer un conocimiento sobre el fenómeno de las

- alternativas terapéuticas. Material y Métodos: investigación descriptiva

- en 250 estudiantes universitarios del área de Ciencias de la Salud que

- firmaron consentimiento informado. Se utilizó como instrumento una

- escala Likert elaborada exprofeso y validada por el método Delphi.

- Se utilizó estadística descriptiva como método de análisis. Resultados:

- se encontró que la actitud de aceptación general frente al uso de

- cannabis medicinal es del 60.4\%; asimismo, el estudio arrojó que los

- universitarios confian en las personas que consumen cannabis para

- tratar enfermedades relacionadas con el dolor y que deben existir

- sitios terapéuticos para tratamiento con cannabis. Conclusiones: el

- dolor crónico constituye uno de los problemas de salud pública más

- relevantes a nivel mundial y tiene un alto impacto en las personas que

- 10 experimentan. Debido a esto, diversas alternativas se presentan

- como opciones terapéuticas y algunas han sido aprobadas para su

- uso por organizaciones mundiales. Ante este panorama, el profesional

- de la salud debe tener conocimiento acerca del fenómeno para poder

- intervenir adecuadamente. LUXMÉDICA AÑO 15, NÚMERO 44, MAYO-

- AGOSTO 2020, PP 3-12.

\section{Abstract}

Objective: To describe the attitude of students of a public university towards the use of cannabis as a treatment for chronic pain, and to know about the phenomenon of therapeutic alternatives. Methods: This is a descriptive research in 250 university students who signed informed consent. A Likert scale elaborated and validated by the Delphi method was used as an instrument. Descriptive statistics were used as the method of analysis. Results: The general acceptance attitude towards the use of medicinal cannabis was $60.4 \%$; also, the study found that university students trust people who use cannabis to treat pain-related illnesses and that there must be therapeutic places for cannabis treatment. Conclusions: Chronic pain is one of the most relevant public health problems worldwide and has a high impact on people who experience it. Because of this, various alternatives are presented as therapeutic options, and some have been approved for use by global organizations. Given this panorama, the health professional must know about the phenomenon to be able to intervene appropriately. LUXMÉDICA AÑO 15, NÚMERO 44, MAYOAGOSTO 2020, PP 3-12.

* Domingo-Martínez Gabriela Lorena, estudiante de la Licenciatura en Enfermería de la Universidad Autónoma de Querétaro Orcid: 0000-0001-9469329. Correo electrónico gabii_dm94@hotmail.com

* Sánchez-Carbajal Karen Daniela, estudiante de la Licenciatura en Enfermería de la Universidad Autónoma de Querétaro. Orcid: 0000-0001-9485520X correo electrónico kdanielacarbajal@gmail.com

** Ruíz-Vargas Nancy Viridiana, Licenciada en Enfermería, candidata a Maestra en Enfermería Orcid: 0000-0002-5602-9238. Correo electrónico nancy. ruiz@uaq.mx

*** Gallegos-Torres Ruth Magdalena, Licenciada en Enfermería, Doctora en Ciencias de la Salud. Orcid: 0000-0001-8034-4089 Correo electrónico isisrmgx@gmail.com

**** Oñate-Romero Juan Manuel, Psicólogo clínico, Maestro en Psicoterapia Psicoanalítica de la Infancia y la Adolescencia Orcid: 0000-0001-8217-1062 Correo electrónico jmor1003@hotmail.com

***** Juárez-Nilo Sarket Gizeh, Licenciada en Enfermería, Especialista en Salud Pública. Orcid: 0000-0002-4212-8622 Correo electrónico sajuni20@ hotmail.com

******Xeque-Morales Ángel, Maestro en Ciencias Orcid: 0000-0001-5867-0267 Correo electrónico equismas@gmail.com

Fecha de recibido: 8 de noviembre 2019

Fecha de aceptación: 28 de febrero 2020

Correspondencia: Dra Ruth Magdalena Gallegos Torres. Facultad de Enfermería. Universidad Autónoma de Querétaro. Cerro de las Campanas s/n, Las Campanas. Código postal 76010 Santiago de Querétaro, Qro. México Teléfono: 4421921200 correo: isisrmgx@gmail.com 


\section{Introducción}

El dolor crónico constituye un problema de salud a nivel mundial. Las personas que presentan dolor son afectadas por patologías de diversa índole, que implican el consumo de múltiples medicamentos a lo largo de su tratamiento. Ante esto, la industria farmacéutica ha desarrollado una amplia gama de medicamentos que pueden coadyuvar a la recuperación de quien lo padece. . $3^{-3}$

Una alternativa que ha tomado interés en la comunidad científica para el manejo del dolor es el uso de plantas de tipo medicinal, entre ellas el cannabis, ya que se han reportado efectos analgésicos eficaces. ${ }^{4-6}$

En este sentido, la Organización Mundial de la Salud señala que diversas posibilidades se presentan como opciones terapéuticas ante el dolor crónico, lo cual indica que las personas recurren a tratamientos que den solución a su problema cuando la medicina alópata no tiene una respuesta, siendo una alternativa el uso del cannabis de forma medicinal. ${ }^{7}$

En dicho contexto, la Organización Panamericana de la Salud, en el artículo "Acerca de la propuesta de utilizar cannabis con fines medicinales", refiere que en la actualidad existe fundamentación válida sólo para apoyar el empleo de la planta en caso de náuseas por quimioterapia anticancerosa y admitir su uso en menores dosis para estimular el apetito en enfermos terminales de SIDA. ${ }^{8-10}$

Entretanto, la American Cancer Society apoya la necesidad para más estudios científicos acerca del cannabis medicinal. En este orden de ideas, en el 2017, se publicó en la revista Deutsches Ärzteblatt International un estudio de varias revisiones sistemáticas y estudios observacionales prospectivos sobre el cannabis para el dolor y la medicina paliativa. ${ }^{11}$

Relacionado con lo anterior, un artículo publicado en el Annals of Palliative Medicine en el 2017 determinó que hay evidencia conflictiva sobre dosis más altas y si éstas proveían mayor analgesia y que, como resultado de la revisión realizada por Medline desde 1975 hasta el 2017, se concluyó que muchos reportes no tenían el poder estadístico necesario para establecer conclusiones y que era definitiva la necesidad de ensayos clínicos con mayor tamaño de muestras. ${ }^{11}$ Así, la posibilidad de utilizar el cannabis con fines terapéuticos es atendida por diversas investigaciones, de tal forma que, por el momento, la información disponible sobre estas posibles indicaciones es escasa. ${ }^{12,13}$

En este sentido, un primer punto de partida antes de establecer un conocimiento sobre el fenómeno, es determinar cuál es la actitud hacia el mismo, entendiéndose la actitud como una evaluación general que las personas realizan sobre un objeto $u$ otras personas de su entorno, las cuales pueden ser favorables o desfavorables, e incluyen los elementos: a) cognitivo, que involucra los pensamientos y creencias de la persona acerca del objeto de actitud; b) afectivo, que agrupa los sentimientos y emociones asociados al objeto 
de actitud; y c) de comportamiento, que recoge las intenciones o disposiciones a la acción, así como los comportamientos dirigidos hacia el objeto de actitud. ${ }^{14,15}$

Derivado de lo anterior, la presente investigación permitió identificar la opinión respecto al uso del cannabis terapéutico, que tienen los profesionales del área de la salud.

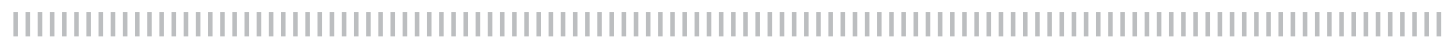

\section{Material y métodos}

Se realizó una investigación descriptiva, transversal y exploratoria, en una muestra de 250 estudiantes universitarios de una universidad pública, del área de Ciencias de la Salud, que cumplieron con los siguientes criterios de selección: ser universitarios inscritos en la Universidad Autónoma de Querétaro, encontrarse en el campus Centro Universitario, estar inscritos en la Licenciatura en Enfermería, de sexo indistinto y aceptar que se les aplicara el instrumento firmando el consentimiento informado; se excluyeron de la investigación aquellos que se negaron a contribuir con la investigación o alumnos que no estuvieran presentes en el momento de aplicar el instrumento. Se descartaron los instrumentos de aquellos participantes que decidían retirarse del estudio o que se encontraran realizando movilidad académica.

Para recabar la información se utilizó un instrumento elaborado ex profeso para la investigación, denominado "Escala Likert de actitud del universitario hacia el uso de cannabis para el tratamiento del dolor crónico", fundamentada en la operacionalización de las variables y en los pasos propuestos por Hernández Sampieri, Fernández Collado y Baptista Lucio. ${ }^{16}$

En este sentido, la operacionalización de las variables fue la siguiente:

\begin{tabular}{|c|c|c|c|c|}
\hline $\begin{array}{l}\text { Variable y tipo de } \\
\text { variable }\end{array}$ & Definición conceptual & Definición operacional & Dimensiones & Escala de medición \\
\hline \multirow{4}{*}{$\begin{array}{l}\text { Variable indepen- } \\
\text { diente } \\
\text { Actitud hacia el uso de } \\
\text { la marihuana }\end{array}$} & \multirow{4}{*}{$\begin{array}{l}\text { Grado con que las personas } \\
\text { tienden a juzgar cualquier as- } \\
\text { pecto de la realidad, pueden ser } \\
\text { positivas, negativas, o neutras y } \\
\text { pueden variar en su extremosi- } \\
\text { dad o grado de polarización }\end{array}$} & \multirow{4}{*}{$\begin{array}{l}\text { Escala Likert: actitud del } \\
\text { universitario hacia el uso de } \\
\text { marihuana para tratamiento } \\
\text { de dolor crónico. }\end{array}$} & Aceptación & Ordinal \\
\hline & & & Creencias & Ordinal \\
\hline & & & Conocimientos & Ordinal \\
\hline & & & Expectativas & Ordinal \\
\hline \multirow{3}{*}{$\begin{array}{l}\text { Variable indepen- } \\
\text { diente } \\
\text { Datos socio-demo- } \\
\text { gráficos }\end{array}$} & \multirow[t]{3}{*}{$\begin{array}{l}\text { Atributos de los sujetos que se } \\
\text { recogen para describir la mues- } \\
\text { tra (Burns y Grove, 2012). }\end{array}$} & \multirow[t]{3}{*}{$\begin{array}{l}6 \text { preguntas: } 3 \text { de respuesta } \\
\text { abierta y el resto con opción } \\
\text { múltiple. }\end{array}$} & Sexo & Nominal \\
\hline & & & Edad & Intervalo \\
\hline & & & $\begin{array}{l}\text { Estado civil } \\
\text { Ocupación } \\
\text { Semestre } \\
\text { Religión }\end{array}$ & Nominal \\
\hline
\end{tabular}


La escala Likert consta de una cédula de datos sociodemográficos que incluyen: sexo, edad, estado civil, religión, ocupación y grado de estudios. Asimismo, contiene cuatro categorías con enfoque en la variable de actitud: 1) Aceptación, 2) Creencias, 3) Conocimientos y 4) Expectativas. Está constituida por 24 preguntas con codificación de ítems que incluye cinco criterios: (1) Totalmente de acuerdo, (2) De acuerdo, (3) Indeciso, (4) Desacuerdo y (5) Totalmente en desacuerdo.

Para la validación del instrumento se utilizó la revisión por expertos, basada en un panel de cinco profesionales del área de la salud y afines, que poseen formación y experiencia en la investigación, que dominan la temática de tratamientos alternativos, adicciones, salud pública, bioestadísti- ca y metodología de la investigación. ${ }^{18}$

El instrumento se envió con la versión final, a cada uno de los revisores expertos en el tema, se realizaron ajustes propuestos por cada uno de ellos y se evaluó nuevamente por medio de pilotaje, para posteriormente realizar las adecuaciones correspondientes. Finalmente se determinó la confiabilidad de la escala Likert de actitud con la prueba Alfa de Cronbach obteniendo un valor de 0.7.

Cabe mencionar que previo a la recolección de datos, se contó con la evaluación y aprobación del proyecto ante el Comité de Investigación y el Comité de Bioética de la Facultad de Enfermería de la Universidad Autónoma de Querétaro, quienes emitieron un dictamen de aprobación.

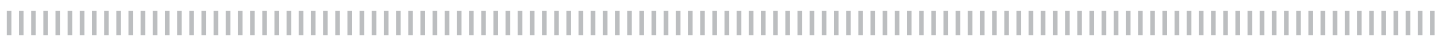

\section{Resultados}

La muestra del estudio estuvo integrada por 250 estudiantes universitarios del área de Ciencias de la Salud de una universidad pública, ubicada en la Ciudad de Querétaro, México. Del total de la muestra, el $90 \%$ fue de sexo femenino, con una media de edad de 19 años (DE 0.92); el $98 \%$ es soltero, el $8.4 \%$ además estudia y trabaja. El $88.8 \%$ de los encuestados es de religión católica.

La categoría de "aceptación", abordada a través de la escala Likert de "actitud", arrojó que los universitarios confían en las personas que consumen cannabis para tratar enfermedades relacionadas con el dolor $(72 \%)$; además, consideran que el consumo de cannabis terapéutico no genera ningún peligro para quien lo consume (62\%); sumado a lo anterior, los estudiantes opinan que deben existir sitios terapéuticos para tratamiento con cannabis (50\%) y el $25 \%$ apoya la idea de contar con personal de salud que trate pacientes con este recurso.

Sin embargo, en la categoría de "creencias" se encontró que tan solo el $26 \%$ de los universitarios considera que consumir cannabis es una gran innovación y opción de medicina natural; no obstante; tan sólo el $10 \%$ considera que el uso de dosis frecuentes de esta sustancia puede producir dependencia y que es muy dañino para todas las personas sin excepción (42\%). De igual forma, el $30 \%$ opina que las personas que consumen cannabis terapéutico le dan mala imagen a la sociedad y hasta el $41 \%$ refiere no estar dispuesto a tener una relación afectiva con alguien que consuma cannabis terapéutico y que aceptar el uso de cannabis terapéutico sería un gran error en la vida de las personas $(41 \%)$; todo lo anterior, sugiere la necesidad urgente de ampliar la investigación enfocada a este recurso terapéutico para poder establecer criterios de fármaco-vigilancia a partir de organismos oficiales, donde además resulta imprescindible estudiar la actitud de aceptación que tiene la sociedad mexicana, pues bien se conoce que este tema está vinculado a aspectos ilícitos.

Continuando con esta descripción, para la categoría de "expectativas", se encontró 
que el $72 \%$ de los universitarios opina que el hecho de que la gente de otros países consuma cannabis terapéutico demuestra que es muy buena opción medicinal. A pesar de ello, el $46 \%$ de los encuestados considera que recomendar cannabis terapéutico puede incrementar la inseguridad en el país, igualmente el $26 \%$ refiere que al legalizar y aceptar el consumo de cannabis se puede incrementar la violencia, lo anterior posiblemente derivado de las características sociopolíticas que experimenta México en materia de tráfico de sustancias. Por otra parte, se observa la opinión de que la aceptación del cannabis terapéutico mejoraría la calidad de vida de muchos pacientes $(35 \%)$, ya que el consumo terapéutico mejora la salud de algunos pacientes (43\%); el $24 \%$ de los encuestados está dispuesto a consumir cannabis terapéutico por recomendación médica, si su precio es accesible $(29 \%)$, y hasta el $50 \%$ de ellos refiere que no le molestaría tener familiares o conocidos que consuman cannabis terapéutico. Con lo anterior se refuerza la importancia de continuar investigando el cannabis desde el abordaje científico.

Finalmente, el resultado global según la escala y las respuestas obtenidas se muestra favorable para los objetivos de esta investigación (figura 1), lo que puede llegar a ser un gran aporte para la medicina alternativa. La tabla 1 muestra el porcentaje de aceptación por cada una de las interrogantes abordadas.

En lo concerniente a describir la actitud del estudiante universitario respecto al uso del cannabis como tratamiento del dolor crónico, que constituyó el objetivo de este estudio, se encontró que la actitud de aceptación general frente al uso de cannabis medicinal es del $60.4 \%$

\section{Gráfica 1. Actitud del universitario hacia el uso del cannabis con fines terapéuticos}

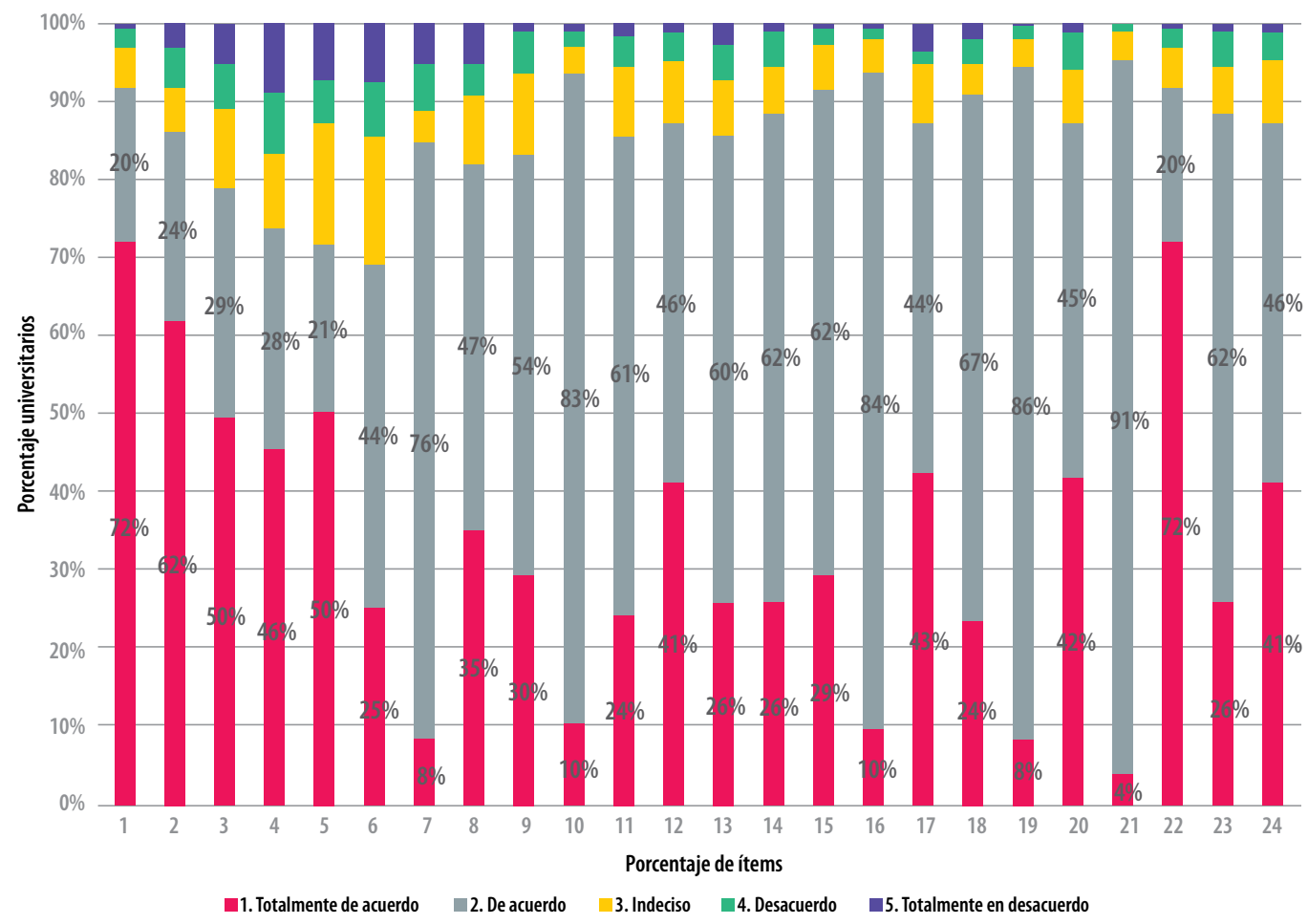

Fuente: Escala Likert de actitud hacia el uso de cannabis, 2018. 


\section{Tabla I}

\section{Porcentaje de respuesta de aceptación de diversas afirmaciones de los estudiantes universita- rios, frente al uso de cannabis medicinal}

\begin{tabular}{|c|c|}
\hline INTERROGANTES & PORCENTAJE DE ACEPTACIÓN \\
\hline $\begin{array}{l}\text { 1. Confío en las personas que consumen cannabis para tratar enfermedades } \\
\text { relacionadas con el dolor. }\end{array}$ & $72 \%$ \\
\hline $\begin{array}{l}\text { 2. Considero que el consumo del cannabis terapéutico no genera ningún peligro para } \\
\text { quien lo consuma. }\end{array}$ & $62 \%$ \\
\hline 3. No me molesta tener familiares o conocidos que consumen cannabis terapéutico. & $50 \%$ \\
\hline 4. El recomendar cannabis terapéutico incrementa la inseguridad en mi país. & $46 \%$ \\
\hline 5. Deberían existir sitios terapéuticos para tratamiento con cannabis. & $50 \%$ \\
\hline 6. Me sentiría a gusto al contar con personal de salud que trate pacientes con cannabis. & $25 \%$ \\
\hline La aceptación de cannabis es de gran utilidad para la medicina natural en mi país. & $8 \%$ \\
\hline $\begin{array}{l}\text { 8. Pienso que la aceptación del cannabis terapéutico mejoraría la calidad de vida de } \\
\text { muchos pacientes. }\end{array}$ & $35 \%$ \\
\hline 9. Las personas que consumen cannabis terapéutico le dan mala imagen a la sociedad. & $30 \%$ \\
\hline 10. Recomendaría el consumo de cannabis terapéutico a mis familiares o conocidos. & $10 \%$ \\
\hline 11. No me molesta interactuar con personas que han consumido cannabis terapéutico. & $24 \%$ \\
\hline $\begin{array}{l}\text { 12. No estoy dispuesto a tener una relación afectiva con alguien que consuma cannabis } \\
\text { terapéutico. }\end{array}$ & $41 \%$ \\
\hline $\begin{array}{l}\text { 13. Creo que legalizar y aceptar el consumo de cannabis terapéutico incrementa } \\
\text { violencia en mi país. }\end{array}$ & $26 \%$ \\
\hline 14. Consumir cannabis es una gran innovación y opción de medicina natural. & $26 \%$ \\
\hline 15. Estoy dispuesto a consumir cannabis terapéutico si su precio es accesible. & $29 \%$ \\
\hline 16. El uso de dosis frecuentes de cannabis puede producir dependencia. & $10 \%$ \\
\hline $\begin{array}{l}\text { 17. El consumo de cannabis terapéutico le hace mucho bien para la salud para ciertas } \\
\text { personas. }\end{array}$ & $43 \%$ \\
\hline $\begin{array}{l}\text { 18. Estoy dispuesto a consumir cannabis terapéutico si me lo recomendara personal } \\
\text { de salud. }\end{array}$ & $24 \%$ \\
\hline 19. Pienso que nunca consumiría cannabis por ningún motivo. & $8 \%$ \\
\hline 20. El cannabis es muy dañino para todas las personas sin excepción. & $42 \%$ \\
\hline 21. Me perturba considerar que las personas consuman cannabis para tratar enfermedades. & $4 \%$ \\
\hline $\begin{array}{l}\text { 22. El hecho de que la gente de otros países consuma cannabis terapéutico, demuestra que } \\
\text { es muy buena opción medicinal. }\end{array}$ & $72 \%$ \\
\hline $\begin{array}{l}\text { 23. Prefiero no aceptar la opinión de personal de salud que recomienda el cannabis } \\
\text { terapéutico. }\end{array}$ & $26 \%$ \\
\hline 24. Aceptar el uso de cannabis terapéutico sería un gran error en la vida de las personas. & $41 \%$ \\
\hline
\end{tabular}

Fuente: Escala Likert de actitud del universitario hacia el uso de cannabis para tratamiento de dolor crónico, 2018.

\section{Discusión}

El potencial terapéutico que se obtiene con las llamadas medicinas alternativas ha inquietado a los profesionales de la salud, pues su uso se ha ampliado en las sociedades. Tal es el caso de quien afirma que el cannabis tiene cierto efecto analgésico,

especialmente para el dolor crónico, esto como consecuencia de la presencia de receptores en las regiones cerebrales que participan en el control de la percepción, tanto a nivel espinal como a nivel supraespinal. ${ }^{19}$ 
Lo anterior concuerda con lo encontrado en la presente investigación, en donde el $72 \%$ de los universitarios opina que el cannabis terapéutico demuestra ser una muy buena opción medicinal, ya que ha sido aceptada en otros países. Igualmente se asocia el limitado acceso a la información respecto a este recurso, ya que los universitarios mencionan que sólo el $10 \%$ recomendaría el consumo del cannabis terapéutico a familiares o conocidos, esto posiblemente es resultado de la escasa información sobre su farmacocinética.

Las exceptivas sobre el uso del cannabis resalta que los estudiantes consideran este principio activo como potencial para su uso ante el dolor crónico, igualmente se menciona que el $50 \%$ de la población está totalmente de acuerdo en que deberían existir sitios terapéuticos para tratamiento con esta sustancia. En este sentido, Peyraube y Bouso describieron en un estudio multinacional, que los pacientes que utilizan cannabis medicinal para el tratamiento de dolor grave y crónico han reportado beneficios; asimismo, exponen que el cannabis tiene menos efectos secundarios que el resto de los fármacos, y que la principal condición médica por la que los pacientes lo empiezan a utilizar es para aliviar el dolor crónico. ${ }^{20,21}$

En lo correspondiente a las creencias, los resultados de este estudio indican que los universitarios conciben que el uso de cannabis puede generar dependencia, así como que el cannabis es dañino en todas las personas sin excepción; sin embargo, el estudio realizado por Galván, Guerrero y Vásquez señala que las creencias sobre el consumo de cannabis se encuentran vinculadas a experiencias personales, información recibida de otras personas, ilusión o deducción; lo cual hace necesario e importante que se hagan estudios que evalúen el sistema de creencias respecto al uso de alternativas de tratamiento. ${ }^{22}$

Por lo anteriormente señalado, se considera pertinente la aproximación a un fe- nómeno de estudio como éste, que pueda ampliar el campo de conocimiento para las ciencias de la salud, pues diversos son los referentes que existen en cuanto al uso de plantas con efectos terapéuticos que desde tiempos remotos en la historia han sido de gran importancia como apoyo a la salud de las sociedades, con gran efectividad, específicamente por su acción terapéutica desinflamante y analgésica, como es el caso del cannabis. ${ }^{23}$

Como resultado de la gran diversidad de fármacos que son utilizados como tratamiento para las personas que sufren de la molesta experiencia del dolor crónico y debido a las múltiples reacciones adversas que experimentan, se hace necesario contar con alternativas de tratamiento que sean eficaces: la más utilizada de acuerdo con los registros en bases de datos son las plantas medicinales. ${ }^{24-26}$

En este sentido, diversas barreras se han encontrado para el uso de plantas con fines terapéuticos, específicamente con el cannabis, y que tienen que ver con las creencias acerca del tema, pues se ha documentado que el desarrollo y uso clínico del cannabis se ha complicado por el reconocimiento del origen botánico. Es por ello por lo que la investigación ha aumentado debido al interés social intenso en la fabricación de medicamentos a base de hierbas disponibles (plantas) de cannabis para uso medicinal. ${ }^{27,28}$

En México, dicha situación se acentúa al tratarse de un tema controversial que ha sido vinculado a aspectos ilícitos. A pesar de ello, en el boletín publicado en diciembre de 2016, el Senado de la República aprobó con 98 votos a favor, siete en contra y una abstención, el dictamen que reforma diversas disposiciones de la Ley General de Salud y del Código Penal Federal, a fin de permitir en el país el uso medicinal del cannabis; es importante mencionar que este resultado sólo fue un censo, ya que la propuesta aún no es totalmente aceptada, pero se mantiene en constante investigación con el fin de 
que se llegue a un acuerdo. ${ }^{29,30}$

Cabe señalar que la National Institute on Drug Abuse (NIDA) menciona que existen avances en el uso del cannabis medicinal, dado que la planta contiene sustancias químicas que son útiles para tratar una am- plia variedad de enfermedades y síntomas, diversas investigaciones sostienen que se debería legalizar su uso con fines medicinales; por lo tanto, cada vez son más los países que han legalizado el cannabis en este sentido. ${ }^{31,32}$

\section{Conclusiones}

Con todo lo anterior, se puede observar que el tema asociado al uso de cannabis con fines terapéuticos es vigente y ha causado la inquietud del gremio científico, con lo cual se ha ampliado la investigación para poder abordar desde una inclinación diversa el tema en cuestión. Tal es el caso de la presente investigación que permitió establecer un punto de partida para conocer la actitud de estudiantes universitarios del área de salud hacia el uso de alternativas de tratamiento para el dolor que otorga el cannabis, esto de acuerdo con los estudios publicados. Con ello se hace evidente que la actitud de aceptación alcanza un porcentaje importante y que vinculado a ello existen diversas creencias y expectativas que guían la actitud de los futuros profesionales.

Esto genera nuevos retos para la comunidad científica y del área de la salud ya que, sea o no apoyado el uso clínico de cannabis, todos los profesionales sanitarios se pueden encontrar con pacientes que opten por utilizar alternativas de tratamiento. Por lo tanto, es inherente contar con información sobre los aspectos clínicos relacionados con el uso de cannabis y otros elementos de la medicina alternativa.

Con todo esto se genera la necesidad de mayor investigación sobre el cannabis para determinar mejor sus efectos a nivel de salud individual y pública, y para avanzar en la comprensión del potencial farmacéutico de los cannabinoides como medicamentos.

\section{Bibliografía}

1. Vilosio, J. El dolor en nosotros. Buenos Aires: Internet, 2015. Disponible en: http://www.fsg.org. ar/20150316-VILOSIO.pdf

2. International Association for the Study of Pain. IASP. Epidemiología del dolor Articular. [Artículo]. Estados Unidos de América. (2017). Recuperado de: https:// www.iasppain.org/files/Content/ContentFolders/ GlobalYearAgainstPain2/2016/FactSheets/Spanish/11_Formatted_Spanish.pdf

3. International Association for the Study of Pain. IASP. Epidemiología del dolor Articular. [Artículo]. Estados Unidos de América. (2016). Recuperado de: https:// www.iasppain.org/files/Content/ContentFolders/ GlobalYearAgainstPain2/2016/FactSheets/Spanish/11_Formatted_Spanish.pdf

4. Reyes, O. El uso terapéutico del cannabis [Grado de Enfermería]. España: Universidad de la Laguna, Facultad de Enfermería y Fisioterapia, 2015. Recuperado de: https://riull.ull.es/xmlui/bitstream/ handle/915/2230/El\% 20uso\% 20terapeutico \% 20 del\% 20Cannabis.pdf?sequence $=1$
5. National Center for Complementary and Alternative Medicine NCCAM. ¿Qué es la medicina complementaria y alternativa? [Institutos Nacionales de la Salud (NIH)]. Departamento de Salud y Servicios Humanos de los Estados Unidos (HHS). (2011). Recuperado de https://nccih.nih.gov/sites/nccam.nih.gov/files/ informaciongeneral.pdf

6. Burguillo, D. Cannabis: Posibilidades Terapéuticas en el ámbito Enfermero. España: Revista Enfermería C y L. (2015). Recuperado de: www.revistaenfermeriacyl.com/index. php/revistaenfermeriacyl/article/.../145/114

7. Organización Mundial de la Salud. OMS. Uso medicinal de la marihuana, riesgos y beneficios. El rol de la regulación. [Pan American Health Organization.] Estados Unidos de América. (2015). https://www. paho.org/hq/index.php?option=com_content\&vie $\mathrm{w}=$ article\&id $=11121$ : seminar-medical-marijuana-ri sk-benefits\&ltemid $=41080 \&$ lang $=e s$

8. Organización Panamericana de la Salud. Acerca de la propuesta de utilizar la marihuana con fines medicinales. [Scielo Public Health]. (2017). Recuperado de:http://www.scielosp.org/scielo.php?script $=$ sci_ arttext\&pid=S102049891997000800009 
9. International Association for the Study of Pain IASP. ¿Cómo se define el dolor crónico? [Grünenthal Group]. América Latina. (2017). Recuperado de: http://www.changepain.org/grtchangepainportal/ change_pain_home/chronic_pain/insight/definition/es_ES/324800317.jsp

10. Coppari. Calidad de vida y afrontamiento en pacientes con cáncer y sida de Paraguay. [Salud \& Sociedad: Investigaciones en Psicología de la Salud y Psicología social]. Paraguay. (2014). Recuperado de: http://pepsic.bvsalud.org/scielo.php?script=sci_artt ext\&pid $=$ S071874752014000100006

11. Blake A, Wan BA, Malek L, DeAngelis C, Diaz P, Lao $N$, et al. A selective review of medical cannabis incancerpainmanagement.AnnPalliatMed.2017;6(Sup pl2):S215-S222.doi: 10.21037/apm.2017.08.05.

12. Codas, M. El cannabis en el dolor crónico ¿una indicación respaldada por evidencia científica? Rev. virtual Soc. Parag. Med. Int. setiembre 2018. Recuperado de: http://revista.spmi.org.py/index.php/ rvspmi/article/view/128/122

13. Martel. Propuesta para la legalización de la marihuana. [Artículo de Opinión]. Palacio de Minería, F.I., UNAM. México. (2016). Recuperado de: https://www.gob.mx/cms/uploads/attachment/ file/85783/3.Cristinne_Leo_Martel.pdf

14. Guil Bozal M. (2006). Escala mixta Likert-Thurstone. Revista andaluza de Ciencias Sociales. 5

15. Petty, R.E. y Wegener, D.T. (1998). Attitude change. En D. Gilbert, S. Fiske, y G. Lindzey (Eds.), The Handbook of Social Psychology (4th ed.). New York: McGraw-Hill. En Briñol P, Horcajo J, Becerra A, Falces C, Sierra B. (2002). Cambio de actitudes implícitas. Psicothema. 14(4). 771-775.)

16. Hernández Sampieri. Metodología de la Investigación. [Quinta Edición]. McGraw-Hill / Interamericana Editores. (2016). Recuperado de: http://www.pucesi.edu.ec/web/wpcontent/ uploads/2016/04/Hern\% C3\% A 1ndez-Sampieri-R.-Fern \% C3\% A1ndezCollado-C.-y-Baptista-Lucio-P.-2003.-Metodolog\% C3\% ADa-delainvestigaci\% C3\%B3n.-M\% C3\% A9xico-McGrawHill-PDF.-Descarga-enl\% C3\%ADnea.pdf

17. Burns $\mathrm{N}$ y Grove S. Investigación en enfermería. [Quinta Edición]. Elsevier Saunders. España (2012).

18. Barraza Macías A. La consulta a expertos como estrategia para la recolección de evidencias de validez basadas en el contenido. Investigación Educativa Duranguense; 2007,7:5-15. Recuperado de:

https://dialnet.unirioja.es/servlet articulo?codigo $=2358908$

19. Peyraube y Bouso. ¿Marihuana como medicina? Usos médicos y terapéuticos del cannabis y los cannabinoides. [Informe]. México Unido contra la Delincuencia, A.C. (2015). Recuperado de: http://www. senado.gob.mx/comisiones/relext_orgint/ungass/ docs/Documentos-portemas/Salud-y-drogas/Informe-Marihuana-como-Medicina-MUCD.pdf

20. Sánchez. Análogos de cannabinoides en el tratamiento del dolor. [Artículo]. Departamento de Química Orgánica y Farmacéutica, Facultad de Farmacia, Universidad Complutense de Madrid, España. (2016). Recuperado de: http://www.analesranf.com/index. php/aranf/article/view/1711/1728

21. Medel Reyes. El significado que le otorgan los enfermos crónicos al uso de cannabis medicinal en su salud. Universidad de Chile. (2016). Recuperado de: http://www.fundaciondaya.org/wp-content/ uploads/2017/08/Tesiscannabis-medicinal-Macarena-Medel.pdf

22. Briñol Actitudes. [Capítulo 17]. Universidad Autónoma de Madrid. (2015). Recuperado de: https://www. uam.es/otros/persuasion/papers/Actitudes.pdf

23. Galván, Gonzalo; Guerrero-Martelo, Manuel; Vásquez De la Hoz, Francisco Cannabis: una ilusión cognitiva Revista Colombiana de Psiquiatría, vol. 46, núm. 2, abril-junio, 2017, pp. 95-102 Asociación Colombiana de Psiquiatría Bogotá, D.C., Colombia. Recuperado de: http://www.redalyc.org/ pdf/806/80650840007.pdf

24. Medicinal cannabis: A primer for nurses. Nursing (2017). Recuperado de https://journals.lww. $\mathrm{com} /$ nursing/fulltext/2017/08000/Medicinal_ cannabis_A_primer_for_nurses.11.aspx

25. Gallegos. Las plantas medicinales: principal alternativa para el cuidado de la salud, en la población rural de Babahoyo, Ecuador. [Anales de la Facultad de Medicina] (2016). Recuperado de: http://www. scielo.org.pe/scielo.php?pid=\$1025558320160004 00002\&script $=$ sci_arttext

26. Urióstegui Flores. Hierbas medicinales utilizadas en la atención de enfermedades del sistema digestivo en la ciudad de Taxco, Guerrero. México. (2015). [Revista de Salud Pública]. Universidad Nacional de Colombia. Bogotá, Colombia. Recuperado de: http:// www.redalyc.org/articulo.oa?id $=42242322008$

27. Alcántara Montero y González Curado Cannabis en el tratamiento del dolor: consideraciones clínicas y de investigación. [Artículo]. Revista de la Sociedad Española del Dolor. (2017). Recuperado de: http:// scielo.isciii.es/scielo.php?script=sci_arttext\&pid $=$ S1134-80462017000100010

28. Universidad Nacional Autónoma de México. Mariguana. [Biblioteca Digital de la Medicina Tradicional Mexicana]. México. (2009). Recuperado de: http:// www.medicinatradicionalmexicana.unam. $\mathrm{mx} /$ termino.php? $\mid=1 \& \mathrm{t}=$ mariguana

29. Velázquez Benítez. Polémica actual a nivel internacional sobre la legalización de la marihuana. Correo Científico Médico, (2017). Recuperado de http:// scielo.sld.cu/scielo.php?script $=$ sci_arttext $\&$ pid $=$ S1560-43812017000100021

30. Diario Oficial de la Federación. Ley General de Salud. (2014) Recuperado de: http://www.diputados.gob. $\mathrm{mx} /$ LeyesBiblio/pdf/142_220617.pdf

31. Rico Borrego. Alternativas Farmacológicas para el tratamiento del dolor crónico no oncológico. Barcelona: Glosa. (2016). Recuperado de http:// www.asociacionandaluzadeldolor.com/wpcontent/uploads/2016/02/alternativas-farmacologicas-2015.pdf

32. National Institute on Drug Abuse; NIDA. La marihuana como medicina. [Institutos Nacionales de la Salud (NIH)]. Estados Unidos de América. (2017). Recuperado de: https://www.drugabuse.gov/es/publicaciones/drugfacts/lamarihuana-como-medicina. 